\title{
Difference in perception of advantages and disadvantages of automated peritoneal dialysis for patients undergoing peritoneal dialysis: a conjoint analysis
}

Hisako Yoshida ${ }^{1,3}$ and Kazuhiko Tsuruya ${ }^{2,3^{*}}$ (1)

\begin{abstract}
Background: In Japan, only less than 3\% of patients undergoing maintenance dialysis select peritoneal dialysis (PD) as the mode of renal replacement therapy. Half of patients undergoing PD use a cycler for automated PD (APD). However, it remains unclear what patients undergoing PD regard as the benefits of APD. The purpose of this study was to objectively clarify what patients undergoing PD regard as the advantages and disadvantages of APD.

Methods: We designed case simulations that varied according to 6 characteristics of PD, such as the adequacy of dialysis and frequency of bag exchange, and created 16 simulation cards for conjoint analysis. For each case vignette, the respondents were asked to indicate whether they would receive this modality of PD on a 5-point scale.

Results: Thirty-two patients undergoing PD were recruited for this study and completed conjoint analysis simulation questionnaires. The factor with the highest importance in evaluating the modality of PD was the frequency of bag exchange among all participants. When participants were divided into those undergoing APD and continuous ambulatory PD (CAPD), the factor was different between them; that is, the low frequency of bag exchange is regarded as an advantage of APD for patients undergoing APD, while the large-sized cycler device is regarded as a disadvantage of APD for those undergoing CAPD.
\end{abstract}

Conclusions: The perception of advantages and disadvantages of APD differed between patients undergoing APD and CAPD; the former focus on the frequency of bag exchange, whereas the latter focused the size of device.

Keywords: APD, CAPD, Conjoint analysis, Cycler

\section{Introduction}

The number of patients with end-stage kidney disease who need renal replacement therapy (RRT) has increased worldwide. Several forms of RRT are available, including hemodialysis, peritoneal dialysis (PD), and renal transplantation. In Japan, however, less than $3 \%$ of patients undergoing maintenance dialysis select PD as the mode

*Correspondence: tsuruya@naramed-u.ac.jp

2 Department of Nephrology, Nara Medical University, 840 Shijo-cho, Kashihara, Nara 634-8521, Japan

Full list of author information is available at the end of the article of RRT [1] despite the various medical and social benefits presented by PD. The medical benefits of PD include the absence of the need for vascular access [2], prolonged preservation of residual renal function [3], and a lower cardiovascular stress due to continuous removal of extracellular fluid [4]. The higher quality of life in comparison with in-center hemodialysis $[5,6]$ is seen as a social benefit of PD. Conversely, because the effective management of PD largely depends on self-care, volume overload is more frequently encountered in patients undergoing PD than among those undergoing hemodialysis. 
Automated PD (APD), performed with a device, makes PD a convenient option. In Japan, nearly $40-50 \%$ of all patients undergoing PD use a cycler device [7]. APD enables frequent exchange of dialysate and reduces the injection volume of dialysate, which can relieve high abdominal pressure. Therefore, even if patients experience complications such as inguinal hernia or pleuroperitoneal communication, they may continue treatment via PD [8-10]. Furthermore, APD is often carried out overnight, allowing the daytime to be free of dialysis treatment; this is more compatible with work, school, and other lifestyle commitments and reduces the burden on caregivers [11-13]. However, some doctors do not recommend APD due to their assumption of a more rapid decrease in residual renal function in APD compared to continuous ambulatory PD (CAPD).

To date, few reports address doctors' or patients' opinions regarding APD. Therefore, we conducted conjoint analysis on data obtained via a questionnaire study of patients undergoing CAPD and APD so as to determine their opinions of APD using conjoint analysis.

\section{Methods}

\section{Participants}

The present study included patients undergoing PD who had been treated at Kyushu University Hospital from June 2008 to August 2008. All patients were treated using the PD system provided by Baxter (Tokyo, Japan), and only one type of APD device (Home APD system "YUME") was used in our PD center. All patients had a good understanding of CAPD and APD including the APD device because of sufficient explanation at the time of PD initiation. These patients were also entered into a PD registrycohort study that was approved by the Kyushu University Institutional Review Board (Approval number: 23-124). Each patient's response to the questionnaire was considered to be their written informed consent. Because this study included patients who had already decided on either CAPD or APD, but not those who were about to undergo decision-making, it was not possible to determine whether the result of this analysis affected the decision-making process.

\section{Conjoint analysis}

Conjoint analysis is used to assess respondents' preferences over a set of multi-attribute alternatives. It was introduced into marketing research in the early the 1970s and has since been used for complex decision-making as well as assessment, comparison, and/or evaluation in both economic and medical research $[14,15]$.

The case simulations used in this study varied according to six characteristics (Table 1). These include: (1) adequacy of dialysis-entailing increased fluid removal and increased solution removal, constant fluid removal and increased solution removal, or increased fluid removal and constant solution removal, (2) abdominal pressure-increased or decreased, (3) risk of peritonitis-increased, decreased, or unchanged, (4) frequency of bag exchange-30 $\min \times 2$ times per day, $30 \mathrm{~min} \times 4$ times per day, or only overnight without any bag exchange during the day, (5) size of the APD device-large, small, or unnecessary, and (6)

Table 1 Variations in the case simulations according to six characteristics

\section{Features of hypothesized treatments}

Medical characteristics

Adequacy of dialysis

Abdominal pressure

Risk of peritonitis

Non-medical characteristics

Frequency of bag exchange

Size of device

Technique

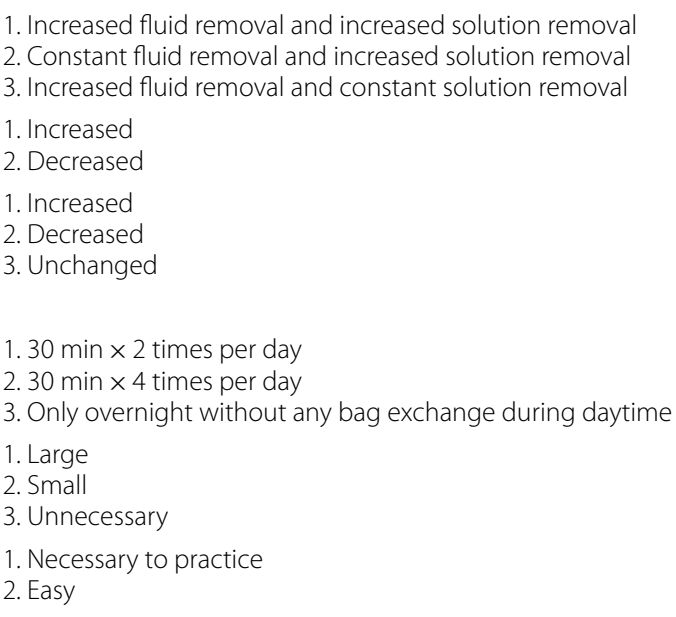

The case simulations varied according to six characteristics 
technique-necessary to practice or easy. We designed 324 written case simulations in combination, making use of each level- $3 \times 2 \times 3 \times 3 \times 3 \times 2$. We then selected 16 case simulations according to an orthogonal table, to ensure the absence of collinearity, and created 16 conjoint cards. An example of a case vignette is presented in Fig. 1. For each case vignette, the respondents were asked to indicate whether they would receive this modality of PD on a 5-point scale. Furthermore, we collected information from the respondents regarding their age, sex, daytime work, current PD modality (APD or CAPD), and PD vintage.

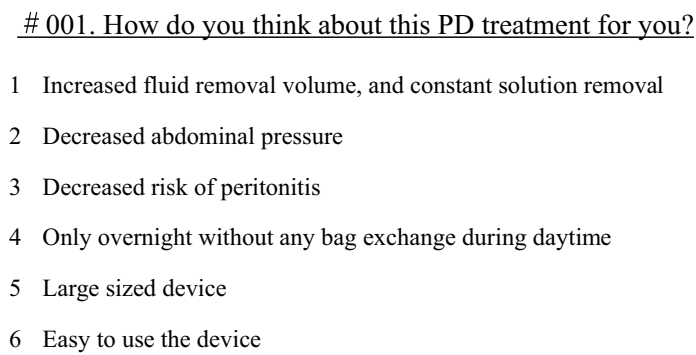

Fig. 1 An example of a simulation card used in the present study. PD, peritoneal dialysis

\section{Statistical analysis}

Data are expressed as mean \pm standard deviation, median [first quartile, third quartile], or number (percentage) unless otherwise specified. The chi-square test, unpaired $t$ test, and Mann-Whitney test were used as appropriate measures to describe the differences between the two groups. Conjoint analysis was conducted to provide the relative importance and partial utility scores. We performed univariable regression analyses to confirm the association of the patients' characteristics with the relative importance score calculated in performing the conjoint analysis. A two-tailed $p$ value of $<0.05$ was considered statistically significant. A conjoint model was conducted using the conjoint-analysis package of SPSS 17.0 (SPSS Inc., Chicago, IL, USA), and all other statistical analyses were performed using $\mathrm{R}$ version 3.2.5 ( $\mathrm{R}$ Foundation for Statistical Computing, Vienna, Austria).

\section{Results}

Of the 32 patients undergoing PD who were included in this study, 21 were male and 9 had diabetes mellitus. The mean PD vintage was 24 months (with a range of, 4-48 months), and 17 patients used APD systems. Table 2 shows the demographic characteristics and information on the current dialysis condition of each participant. The mean PD vintage and median dialysate volume were significantly higher in patients undergoing APD than in those undergoing CAPD $(p<0.030$ and $p<0.004$, respectively). Patients undergoing APD were significantly

Table 2 Clinical features of all participants

\begin{tabular}{|c|c|c|c|c|}
\hline & Overall $(n=32)$ & CAPD $(n=15)$ & APD $(n=17)$ & $p$ value \\
\hline Male sex & $21(65.6)$ & $9(60.0)$ & $12(70.6)$ & 0.529 \\
\hline Age, years & $57 \pm 13$ & $63 \pm 12$ & $51 \pm 11$ & 0.006 \\
\hline Dialysis vintage, months & $24 \pm 13$ & $19 \pm 13$ & $29 \pm 12$ & 0.030 \\
\hline Diabetic nephropathy & $9(28.1)$ & $4(26.7)$ & $5(29.4)$ & 0.863 \\
\hline Icodextrin & $28(87.5)$ & $14(93.3)$ & $3(17.7)$ & 0.603 \\
\hline $2.5 \%$ dialysate & $7(21.9)$ & $4(26.7)$ & $3(17.7)$ & 0.678 \\
\hline UV connection device & $4(12.5)$ & $4(26.7)$ & $0(0.0)$ & 0.038 \\
\hline Dialysate volume, L/day & $6.0[6.0,8.3]$ & $6.0[4.5,6.0]$ & $8.1[6.0,9.4]$ & 0.004 \\
\hline D/P creatinine ratio & $0.695[0.580,0.809]$ & $0.636[0.558,0.805]$ & $0.744[0.618,0.847]$ & 0.336 \\
\hline Residual Kt/V weekly & $0.464[0.256,0.840]$ & $0.474[0.285,0.841]$ & $0.382[0.079,0.827]$ & 0.610 \\
\hline Total Kt/V weekly & $1.722[1.528,1.953]$ & $1.713[1.614,1.914]$ & $1.730[1.505,1.970]$ & 0.664 \\
\hline History of peritonitis & $7(21.9)$ & $4(26.7)$ & $3(17.6)$ & 0.678 \\
\hline $\mathrm{BMI}, \mathrm{kg} / \mathrm{m}^{2}$ & $22.7 \pm 2.8$ & $22.5 \pm 2.8$ & $22.8 \pm 2.9$ & 0.801 \\
\hline $\mathrm{SBP}, \mathrm{mmHg}$ & $133.4 \pm 16.0$ & $136.7 \pm 13.8$ & $130.4 \pm 17.6$ & 0.272 \\
\hline $\mathrm{DBP}, \mathrm{mmHg}$ & $77.8 \pm 16.0$ & $81.0 \pm 16.9$ & $74.9 \pm 15.2$ & 0.289 \\
\hline Works in daytime & $18(56.3)$ & $7(46.7)$ & $11(64.7)$ & 0.477 \\
\hline
\end{tabular}

Data are presented as mean \pm standard deviation, $n(\%)$, or median [first quartile, third quartile]

Bold font indicates significant $p$ value $(p<0.05)$.

APD, automated peritoneal dialysis; BMI, body mass index; CAPD, continuous ambulatory peritoneal dialysis; DBP, diastolic blood pressure; D/P, dialysate-to-plasma; SBP, systolic blood pressure; UV, ultraviolet 
younger than those undergoing CAPD $(p<0.006)$. No patients in the APD group used an ultraviolet connection device. The rate of peritonitis history was not significantly different between the two groups ( $p=0.678$ ).

We calculated the relative importance, indicating the strength of the influence of the participants' judgment (Fig. 2a). The factor of the highest importance in evaluating the modality of PD among the participants was the frequency of bag exchange, followed by the size of the APD device and the risk of peritonitis. The technique used to operate the PD assist device was of minimum importance among the six categories in this study. APD, as the current PD modality, positively influenced the frequency of bag exchange and negatively influenced the size of the device. Figure $2 b$ shows the comparison between the current modalities after adjustment for age and sex. Although the frequency of bag exchange had the highest importance for patients undergoing APD, the size of the APD device had the highest importance for patients undergoing CAPD. The difference in the importance of the size of the device between the CAPD and APD groups remained significant, while there was no significant difference in the frequency of bag exchange, after adjusting for age and sex. These results were maintained even if after adjusting for the history of peritonitis, dialysis vintage, UV connection device, and dialysis volume (data not shown). The utility score means the weight of each alternative (element) for decision-making as part worth. The total of the scores of alternatives within each factor becomes zero. The utility scores of all levels in each category are shown in Fig. 3a. In comparison with current modality (CAPD vs. APD), low frequency of bag exchange and freedom during the daytime positively affected the judgment of patients undergoing APD, but a

(a)

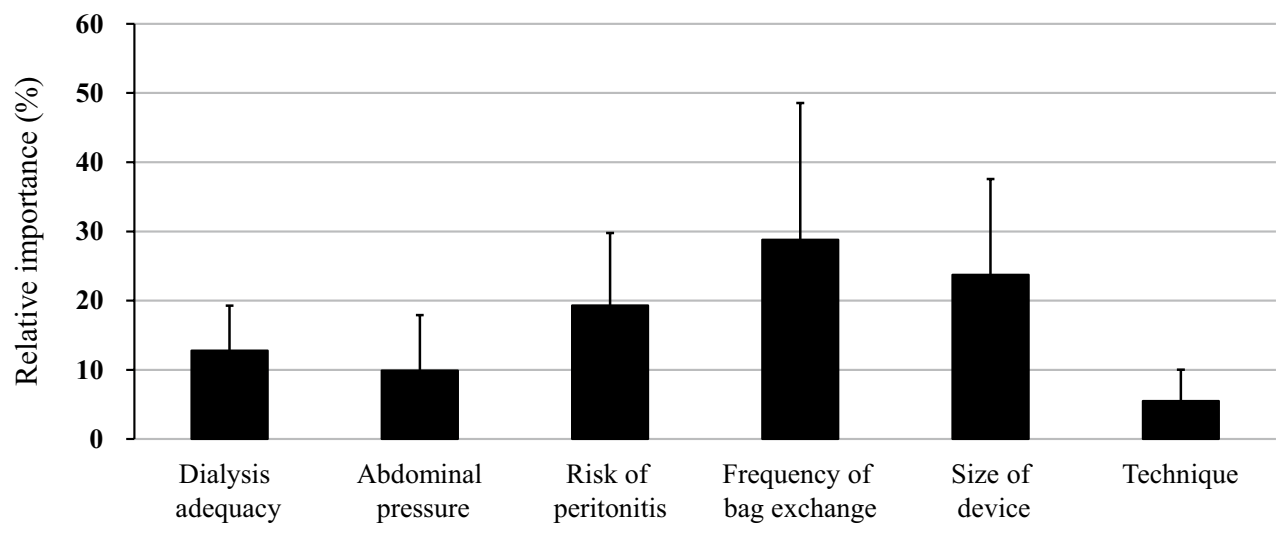

Medical factors

Non-medical factors

(b)

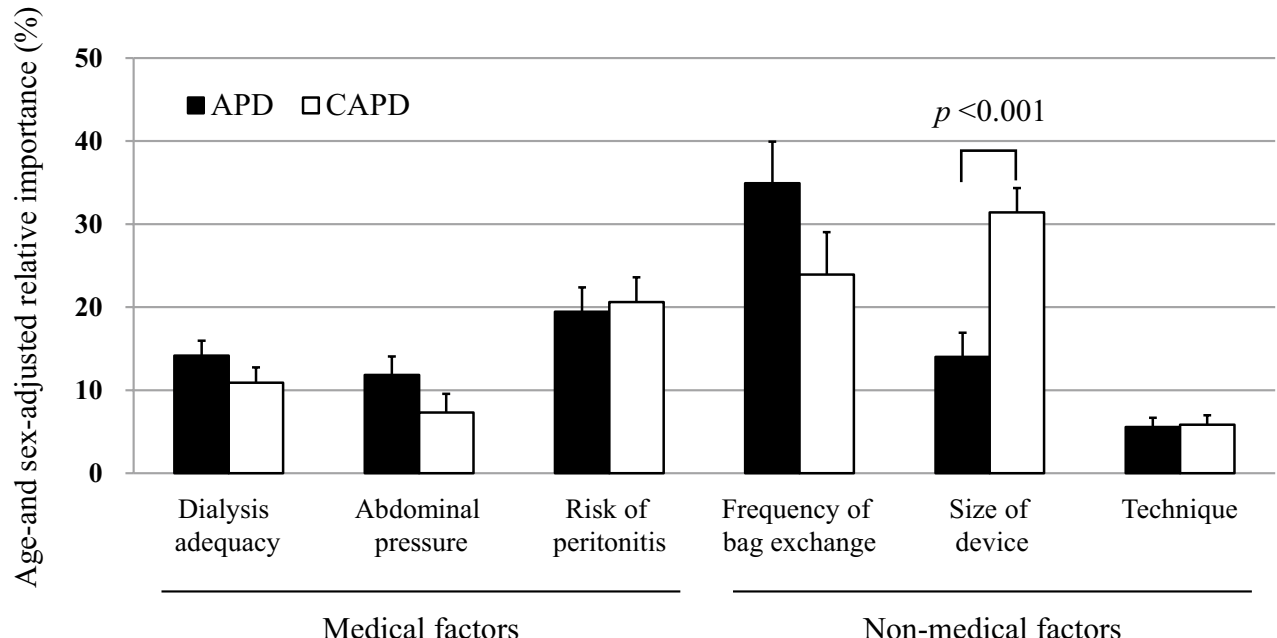

Fig. 2 Relative importance of peritoneal dialysis modalities. a All patients. b Patients classified by current modality; The data of patients undergoing APD and CAPD are shown in closed and open bars, respectively. APD, automated peritoneal dialysis; CAPD, continuous ambulatory peritoneal dialysis 
(a)

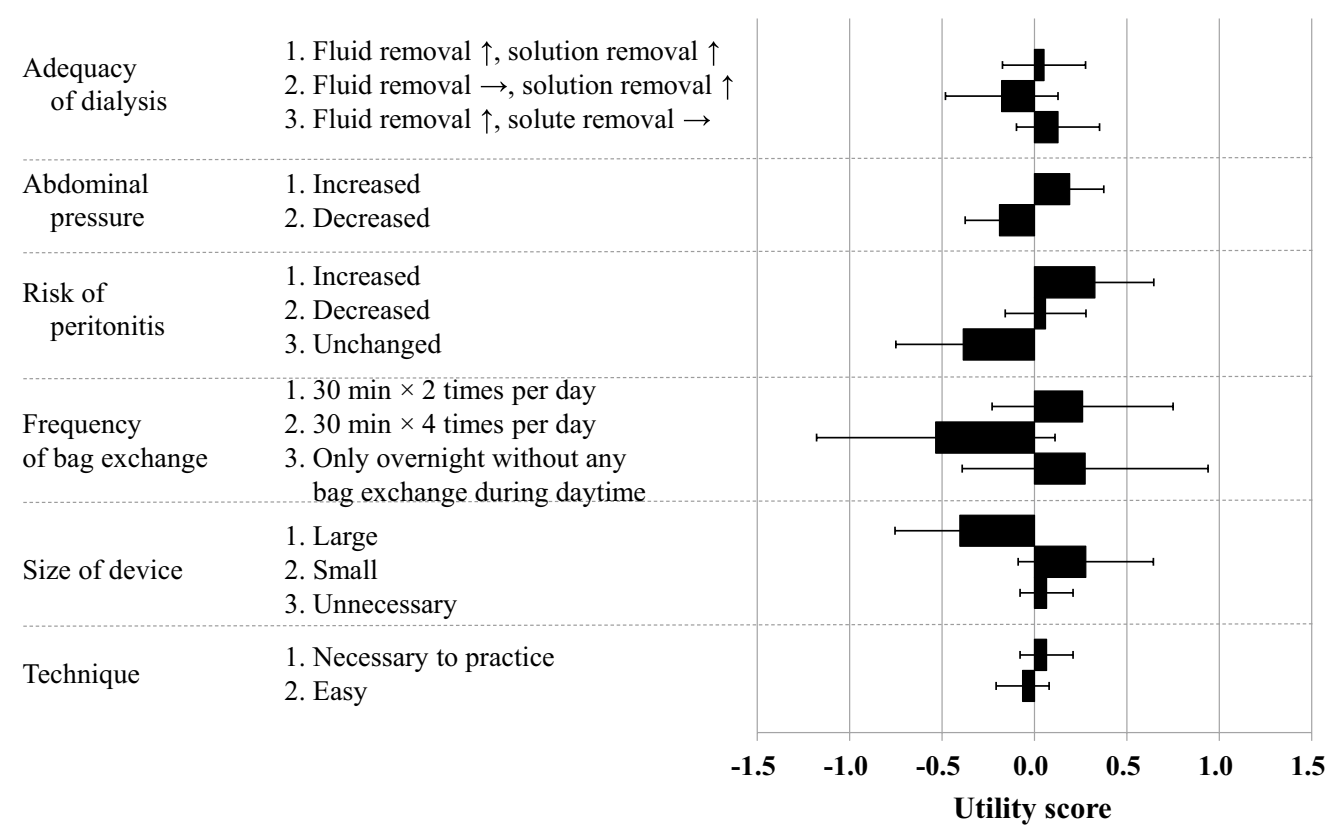

(b)

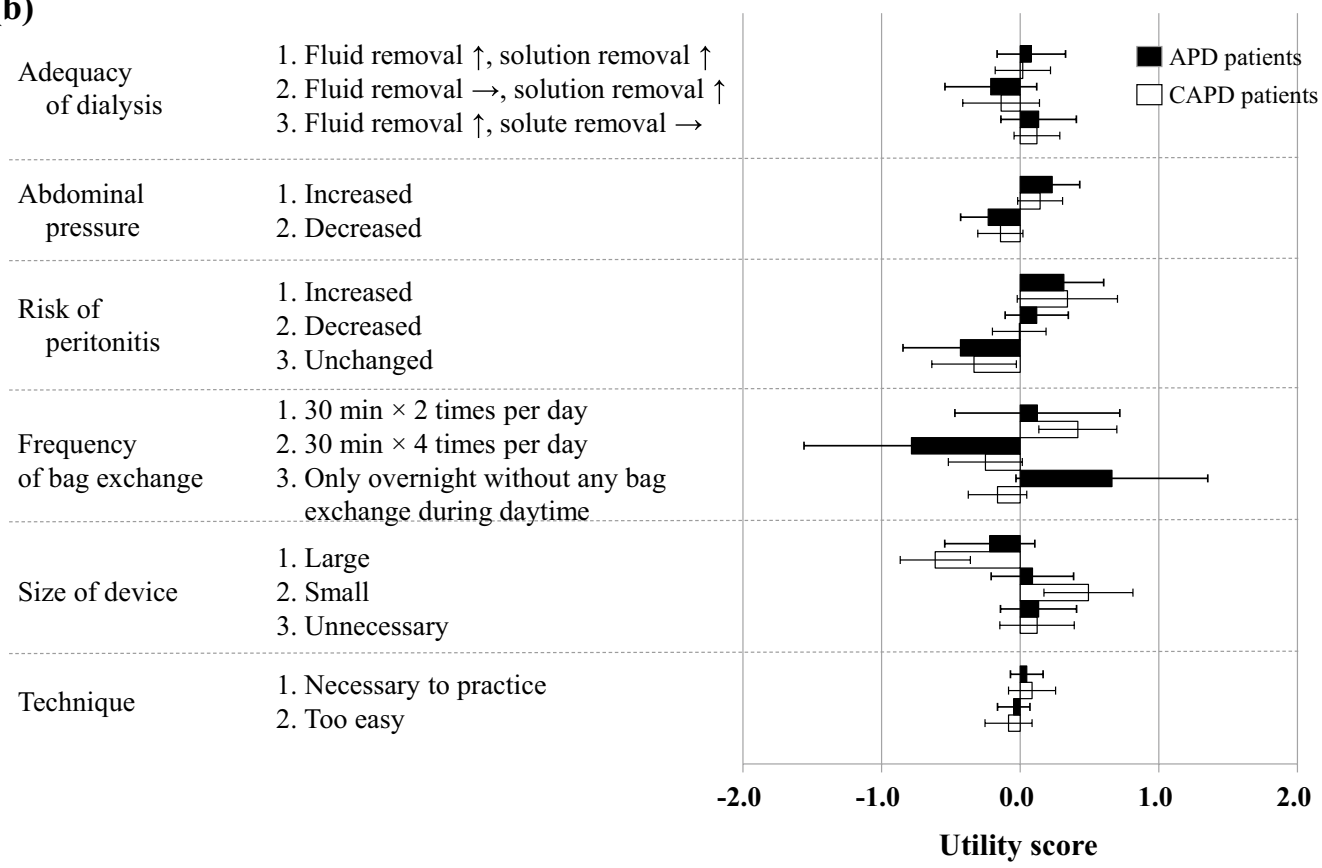

Fig. 3 Utility scores for opinions regarding PD modalities. Data are shown as the least square mean \pm standard error. a All patients. $\mathbf{b}$ Patients classified by current modality; the data of patients undergoing APD and CAPD are shown in closed and open bars, respectively. APD, automated peritoneal dialysis; CAPD, continuous ambulatory peritoneal dialysis

small-sized assist device positively affected the judgment of patients undergoing CAPD (Fig. 3b). All utility scores were calculated and compared between the current PD modality (CAPD and APD) used by the participants (Additional file 1: Table S1).

\section{Discussion}

We examined the advantages and disadvantages of APD among patients undergoing PD using a conjoint analysis method. The factor with the highest importance in evaluating the modality of PD was the frequency of 
bag exchange among all participants, but was different between patients undergoing APD and CAPD; that is, the low frequency of bag exchange is regarded as an advantage of APD for patients undergoing APD, while the large-sized cycler device is regarded as a disadvantage of APD for those undergoing CAPD. One may point out that most patients studied had long-term stable PD treatment and were satisfied with CAPD or APD treatment, respectively, which may have influenced the results of this study. The present study is the first to quantify the opinions of patients undergoing PD with regard to PD modality.

Kim et al. reported that residual renal function declined more rapidly in patients who started dialysis on APD than those who started dialysis on CAPD, during the first year after the initiation of PD [16], while another study showed no difference between patients on APD and CAPD [17]. Some facilities recommend CAPD without an APD cycler as the first-choice treatment in order to avoid early reduction in residual renal function, although the harmful effect of APD on preservation of residual renal function has not yet been established. However, many patients prefer APD therapy in our hospital because quite a few of our patients work during the daytime; in fact, about half of the patients selected APD therapy since they started PD therapy as their first form of RRT.

Meanwhile, regarding the risk of peritonitis, the effectiveness in prevention of peritonitis by APD remains controversial. APD was not associated with a lower peritonitis rate than CAPD in a large observational study [18]. In contrast, patients on APD were found to have significantly lower rates of peritonitis compared to those on CAPD by meta-analysis [19]. APD implementation requires one connection and one disconnection on a daily basis. Correspondingly, an average of 4 connections and 4 disconnections are required for CAPD. It is obvious that the smaller number of maneuvers required for APD could lead to a significant reduction of peritonitis incidence. More recently, we have also reported a reduced risk of peritonitis in patients on APD compared with those on CAPD in 285 incident PD patients using an observational study with a propensity score method [20].

The main strength of the present study is that it is the first report to investigate the priority of the APD modality considered by PD patients. The conjoint method has enabled us to clarify the importance of each feature of CAPD and APD, relatively rather than absolutely. The participants of this study valued non-medical, rather than medical factors. Although there are many reports comparing the characteristics of APD and CAPD [8-13], few reports on how the advantages and disadvantages of APD affect patients' decision-making are found [21]. We do consider that non-medical factors associated with real-world clinical practices for patients receiving PD are more easily recognized than medical factors in those patients. This result might suggest that our patients did not feel that the adequacy of dialysis and the control of abdominal pressure were factors which needed to be improved upon. Interestingly, the point of preference for the PD modality differed between patients undergoing APD and CAPD. Patients undergoing APD prioritize freedom during the daytime. In contrast, patients undergoing CAPD placed priority on a smaller-sized device.

In contrast, there are some limitations in this study. First, this study included patients who had already decided on either CAPD or APD, but not those who were about to undergo decision-making. Thus, it was not possible to determine whether the result of this analysis affected patients' decision-making. Second, all participants only had experience with their current modality, since we excluded patients who had changed their modality (e.g., APD to CAPD, CAPD to APD). Thus, the results of this study might reflect the patients' satisfaction with the current PD modality instead of their preference for a particular PD modality. This is a limitation of conjoint analysis; it is difficult to imagine a situation that has not yet been experienced. In our hospital, all patients who select the bag exchange modality have seen an APD cycler at least once. Several patients might be affected by the cycler's size. Because only one type of device of one PD company used in this PD center, we could not be possible to compare devices from multiple companies. The APD and UV device of each PD company have improved over this decade; future studies should include patients who are newly about to undergo PD treatment. Third, the small number of participants from a single center is raised. In this point, we calculated the minimum sample size required for conjoint analysis [22]. According to this equation, the sample size for the present conjoint model was 17. Thus, the number of participants in the present study was adequate for calculating the importance and utility scores in the conjoint analyses. However, we hope to compare and confirm the influence of several confounding factors and therefore plan to perform a multicenter study with a large number of patients in the future.

\section{Conclusion}

The perception of advantages and disadvantages of APD differed between patients undergoing APD and CAPD; the former focused the frequency of bag exchange and the latter focused on the size of the device. 


\section{Supplementary Information}

The online version contains supplementary material available at https://doi. org/10.1186/s41100-021-00368-2.

Additional file 1. Table S1 Utility scores according to the current PD modality used by the participants.

\section{Acknowledgements}

We thank Angela Morben, DVM, and ELS, from Edanz Group (www.edanzediti ng.com/ac) and Editage (www.editage.com) for English language editing.

\section{Authors' contributions}

$\mathrm{HY}$ and KT contributed to the study design, acquisition of data, statistical analysis, and interpretation of data. Each author contributed important intellectual content during the drafting of the manuscript and revisions thereof and accepts accountability for the overall work by ensuring that questions pertaining to the accuracy or integrity, of any portion of the work are appropriately investigated and resolved. All authors read and approved the final manuscript.

\section{Funding}

None.

\section{Availability of data and materials}

The datasets used and/or analyzed during the current study are available from the corresponding author upon reasonable request.

\section{Declarations}

\section{Ethics approval and consent to participate}

All procedures performed in studies involving human participants were conducted in accordance with the ethical standards of the Institutional Research Committee at which the studies were conducted (approval number of the Ethics Committee of Kyushu University Hospital: 23-124) and with the 1964 Helsinki declaration and its later amendments or comparable ethical standards. This is a sub-analysis of our multicenter cohort study (Fukuoka Peritoneal Dialysis Database Study), which was registered in the University Hospital Medical Information Network (UMIN) clinical trial registry (UMIN000018902).

\section{Consent for publication}

Not applicable.

\section{Competing interests}

$\mathrm{KT}$ has received honoraria and grants from Baxter Limited. The Department of Integrated Therapy for Chronic Kidney Disease, Graduate School of Medical Sciences, Kyushu University, is an endowment department, supported by grants from Baxter Limited.

\section{Author details}

'Department of Medical Statistics, Osaka City University Graduate School of Medicine, Osaka, Japan. ${ }^{2}$ Department of Nephrology, Nara Medical University, 840 Shijo-cho, Kashihara, Nara 634-8521, Japan. ${ }^{3}$ Department of Integrated Therapy for Chronic Kidney Disease, Graduate School of Medical Sciences, Kyushu University, Fukuoka, Japan.

Received: 4 April 2020 Accepted: 29 August 2021

Published online: 10 September 2021

\section{References}

1. Masakane I, Nakai S, Ogata S, Kimata N, Hanafusa N, Hamano T, et al. An overview of regular dialysis treatment in Japan (as of 31 December 2013). Ther Apher Dial. 2015;19:540-74.

2. Nakamura-Taira N, Muranaka Y, Miwa M, Kin S, Hirai K. Views of Japanese patients on the advantages and disadvantages of hemodialysis and peritoneal dialysis. Int Urol Nephrol. 2013;45:1145-58.
3. Jansen M, Hart A, Korevaar J, Dekker FW, Boeschoten EW, Krediet RT, for the NECOSAD study group. Predictors of the rate of decline of residual renal function in incident dialysis patients. Kidney Int. 2002;62:1046-53.

4. Kuriyama S. Peritoneal dialysis in patients with diabetes: are the benefits greater than the disadvantages? Perit Dial Int. 2007;27(Suppl 2):S190-195.

5. Cameron JI, Whiteside C, Katz J, Devins GM. Differences in quality of life across renal replacement therapies: a meta-analytic comparison. Am J Kidney Dis. 2000;35:629-37.

6. Boateng EA, East L. The impact of dialysis modality on quality of life: a systematic review. J Ren Care. 2011;37:190-200.

7. Hasegawa T, Nakai S, Moriishi M, Ito Y, Itami N, Masakane I, et al. Peritoneal Dialysis Registry with 2012 survey report. Ther Apher Dial. 2015;19:529-39.

8. Enoch C, Aslam N, Piraino B. Intra-abdominal pressure, peritoneal dialysis exchange volume, and tolerance in APD. Semin Dial. 2002;15:403-6.

9. Li X, Ren H, Xie J, Huang XM, Zhang CY, Chen N. Automated peritoneal dialysis is suitable for polycystic kidney disease patients with end stage renal disease. Case Rep Nephrol Dial. 2015;5:140-4.

10. García-Maldonado B, Guerrero-Ortiz M, Gómez-Fuentes JR, GarófanoLópez R, Prados-Soler MC, del Pino y Pino MD. Pleuroperitoneal communication in peritoneal dialysis patient. Nefrologia. 2012;32:551-2.

11. Michels WM, van Dijk S, Verduijn M, Le Cessie S, Boeschoten EW, Dekker FW, et al. Quality of life in automated and continuous ambulatory peritoneal dialysis. Perit Dial Int. 2011;31:138-47.

12. Balasubramanian G, McKitty K, Fan SL. Comparing automated peritoneal dialysis with continuous ambulatory peritoneal dialysis: survival and quality of life differences? Nephrol Dial Transplant. 2011;26:1702-8.

13. Griva K, Goh CS, Kang WC, Yu ZL, Chan MC, Wu SY, et al. Quality of life and emotional distress in patients and burden in caregivers: a comparison between assisted peritoneal dialysis and self-care peritoneal dialysis. Qual Life Res. 2016:25:373-84.

14. Bouma BJ, van der Meulen JHP, van den Brink RBA, Smidts A, Cheriex EC, Hamer HP, et al. Validity of conjoint analysis to study clinical decision making in elderly patients with aortic stenosis. J Clin Epidemiol. 2004;57:815-23.

15. Clark MD, Szczepura A, Gumber A, Howard K, Moro D, Morton RL. Measuring trade-offs in nephrology: a systematic review of discrete choice experiments and conjoint analysis studies. Nephrol Dial Transplant. 2018;33:348-55.

16. Kim $\mathrm{CH}$, Oh HJ, Lee MJ, Kwon YE, Kim YL, Nam KH, et al. Effect of peritoneal dialysis modality on the 1-year rate of decline of residual renal function. Yonsei Med J. 2014:55:141-8.

17. Michels WM, Verduijn M, Grootendorst DC, le Cessie S, Boeschoten EW, Dekker FW, Krediet RT, NECOSAD study group. Decline in residual renal function in automated compared with continuous ambulatory peritoneal dialysis. Clin J Am Soc Nephrol. 2011;6:537-42.

18. Nessim SJ, Bargman JM, Austin PC, Nisenbaum R, Jassal SV. Predictors of peritonitis in patients on peritoneal dialysis: results of a large, prospective Canadian database. Clin J Am Soc Nephrol. 2009;4:1195-200.

19. Rabindranath KR, Adams J, Ali TZ, Daly C, Vale L, Macleod AM. Automated vs continuous ambulatory peritoneal dialysis: a systematic review of randomized controlled trials. Nephrol Dial Transplant. 2007;22:2991-8.

20. Kokubu M, Matsui M, Uemura T, Morimoto K, Eriguchi M, Samejima K, et al. Relationship between initial peritoneal dialysis modality and risk of peritonitis. Sci Rep. 2020;10:18763.

21. Winterbottom A, Bekker HL, Conner M, Mooney A. Choosing dialysis modality: decision making in a chronic illness context. Health Expect. 2014;17:710-23.

22. Orme B. Getting started with conjoint analysis: strategies for product design and pricing research. 2nd ed. Madison: Research Publishers LLC; 2010. p. 64-5

\section{Publisher's Note}

Springer Nature remains neutral with regard to jurisdictional claims in published maps and institutional affiliations. 